\title{
Current Status of Lymphatic Mapping with Sentinel Lymph Node Biopsy (SLNB) in Cutaneous Melanoma
}

\author{
José Francisco Gallegos-Hernández*
}

Department of Head, Neck and Skin Tumors, Oncology Hospital, CMN SXXI, IMSS, Mexico City, Mexico

Received for publication: 10 November 2016; accepted for publication: 25 February 2017

Available online: 4 August 2017

Sentinel lymph node biopsy (SLNB), a procedure known as lymphatic mapping with sentinel lymph node biopsy ${ }^{1}$, was developed, described and published by Donald Morton and Alistair Cochran in $1992^{2}$. It is based on Cabañas' early works in patients with penile cancer, and is current standard procedure for the staging of patients with cutaneous melanoma with Breslow depth greater than $1 \mathrm{~mm}$ without palpable lymph nodes in the lymphatic drainage zone ${ }^{3}$. It is one of the most important advances in oncologic surgery in the last decades: not only did it put an end to the existing controversy to decide between elective and therapeutic dissection in intermediate thickness melanoma, but its implications have been such, that the technique is already essential for lymph node staging in many other neoplasms, especially breast cancer, non-melanoma skin cancer and head and neck mucosal cancer ${ }^{3}$.

It is a minimally-invasive technique that, although not without complications, these are obviously less common compared with those occurring in patients undergoing elective lymph node dissection thus allowing, with a lower rate of complications, to classify patients with stage I and II cutaneous melanoma by risk based on the presence or absence of subclinical (not previously identified) lymph node metastases.

However, for lymphatic mapping and SLNB to be useful and meet its purpose, and for staging to be real, it has to meet some requirements; at least 5:

1. Taking care of technical aspects that enable a high rate of sentinel lymph node (SLN) identification and minimal false negative rate.

2. Low rate of complications.
3. Adequate histological assessment, including immuno= histochemistry.

4. Cautious interpretation of the study sensitivity.

5. Adequate selection of patient candidates for the pro cedure, including the diagnostic procedure.

The technique lymphatic mapping with SLNB is carried out with is essential to ensure adequate identification of the first node that drains the tumor. The combined technique (dye and radiocolloid) is with no doubt the standard procedure and has to be carried out during the primary tumor resection. If the pigmented lesion has been previously split, the biopsy should not be broad and no reconstruction of the area should be made (flaps or grafts).

The pursued objectives with an adequate biopsy and mapping technique are to obtain a high identification rate and a minimal false negative rate.

Currently, SLN accepted identification rate is $99.4 \%$, and false-negative rate depends on primary tumor Breslow leyel, with $4.8 \%$ in patients with intermediate melanoma and $10.5 \%$ in those with thick melanoma, based on the results obtained in D. Morton's MSLT- 1 trial $^{4}$.

The critical points to be followed for an adequate identification of the true SLN are: the first station lymph node zone must be free of palpable lymph nodes; it is advisable to have an ultrasound not showing lymph nodes with suspected metastasis and, if this is the case, ultrasound-guided fine aspiration biopsy should be performed; biopsies with broad margins, with grafts or with flap rotation of any kind should be avoided; use of perilesional colloidal rhenium sulfide on the eve of the intervention or even two hours prior to the

*E-mail for correspondence: jfgalh61@gmail.com (J.F. Gallegos Hernández) 
procedure; performance of preoperative scintigraphy (bidimensional image), which allows for anatomic localization (tridimensional identification can be made with SPECT-CT, especially at sites with complex drainage such as the head and neck), which allows for the drainage site to be identified on the skin; use of perilesional dye at least 10 minutes prior to the procedure and once the incision on the lymph node area is made; and intraoperative palpation of it to rule out by touch the presence of suspicious lymph nodes that have not received the colloid or dye (Dr. Merrick Ross verbal communication). Following these steps enables the highest identification rate possible.

The false negative rate is minimized by following the above steps and adding an adequate histopathological study that allows for sub-microscopic metastases to be identified. Avoiding the study with frozen sections is recommended and waiting for definitive assessment. Negative SLNs with conventional testing (hematoxylin and eosin staining) should be analyzed with immunohistochemical staining (HMB-45 and/ or Melan-A).

The probability of having a metastatic SLN ranges from $5 \%$ to up to $40 \%$ and depends mainly on Breslow's level and, although other factors have been associated, these are not yet clearly defined and are the subject of controversy; these include Clark's level, anatomic site (higher risk in head and neck melanomas), primary tumor regression, ulceration (probably the second most important factor after tumor thickness), limphovascular invasion, mitotic index (in those with Breslow's level lower than $1 \mathrm{~mm}$ ) and age (it is metastatic more frequently in subjects younger than 20 years and it rarely contains metastases in those older than 80 years $)^{5}$.

Both retrospectively and, recently, prospectively ${ }^{4}$, the prognostic value of SLN has been confirmed and SLN histological status is therefore an independent factor, just as tumor thickness. This way, in patients with $1.2 \mathrm{~mm}$ or higher Breslow melanomas, survival is lower, and disease-free interval is shorter in patients with metastatic SLN when compared with those whose SLN is negative for metastasis.

On the other hand, SLN therapeutic value is poor, i.e., the possibility for lymphatic mapping and SLNB to prevent neoplastic progression in patients with metastatic SLN is questionable. In the MSLT-1 trial, survival was not better in patients undergoing SLN biopsy compared with those who remained under observation, and disease-free interval was only $7 \%$ better in intermediate melanomas and $10 \%$ in thick melanomas, i.e., patients undergoing lymphatic mapping and SLNB have a lower relapse index at the mapped zone compared with those who remain under observation.

In patients with metastasis-positive SLN, the number, as well as the size and site of metastasis are prognostic factors for recurrence and survival.

Patients with thin melanomas of less than $1 \mathrm{~mm}$ Breslow thickness have very low possibilities of metastasis to the SLN, 5.2\% on average, and lymphatic mapping with SLNB is therefore not indicated. However, there are subgroups where the procedure should be considered. Probably the factor that most accurately predicts the presence positive SLN in melanomas with less than $1 \mathrm{~mm}$ thickness is tumor thickness: mean SLN metastasis in patients with less than $0.75 \mathrm{~mm}$ Breslow thickness is $2.9 \%$, and in those with 0.75 $\mathrm{mm}$ to $1 \mathrm{~mm}$ it is $7.1 \%$.
Other factors that influence and increase the probability of metastatic SLN in patients with thin melanomas include mitotic index higher than 1 mitosis $/ \mathrm{mm}^{2}$, lymphovascular invasion, ulceration (infrequent in thin melanomas), tumor lymphocytic infiltration and regression, with all these being factors that have to be taken into account when deciding between lymphatic mapping with SLNB or surveillance. For some authors, the possibility of metastatic SLN in the presence of at least one of the above mentioned factors is as high as 18\%:

Unlike intermediate melanomas, the prognosis is not changed by SLN histological status in patients with melano mas with less than $1 \mathrm{~mm}$ Breslow thickness.

In patients with metastasis to the SLN, worldwide consensus is to perform complementary lymphadenectomy of the area regardless of the type of metastasis, although fac tors such as the number of metastatic SLNs, the presence of parenchymal and non-subcapsular metastases, ulceration and tumor thickness are currently known to be directly proportionally associated with the possibility of metastat:ic non-sentinel lymph nodes. However, metastatic SLN-related complementary lymphadenectomy current value lies in that an average of $21 \%$ of these patients will have metastatic lymph nodes at dissection, which allows for them to be stratified by recurrence risk; i.e., those with metastasis in non-sentinel lymph nodes have worse survival and disease-free interval than those with metastases restricted to the SLN and eventually might be candidates for adjuvant therapies or clinical trials.

In summary, lymphatic mapping with SLNB has been instituted as the standard approach for lymph node staging in patients with cutaneous melanoma with Breslow thickness of more than $1 \mathrm{~mm}$. If there is no possibility to perform it, there are two attitudes that can be adopted: to keep the lymph node zone under surveillance or to refer the patient to centers or groups that do it with adherence to international standards (this attitude is preferred).

Lymphatic mapping and SLNB is not indicated in stage 0 or less than $0.75-\mathrm{mm}$ thick melanomas or in those thin melanomas between 0.75 and $1-\mathrm{mm}$ thickness, except if in the patient other risk factors coexist, although these are not yet adequately established. The key to attain success is adhering to the recommended technique since the diagnostic process.

Finally, evidence shows that in patients with metastatic SLN the behavior to be followed is to complete the lymphad-o enectomy of the lymph node area undergoing mapping.

\section{REFERENCES}

1. Gallegos JF ¿Qué es el ganglio centinela? Concepto y aplicaciones en Oncología. Acta Med Gpo Angeles. 2005;3:91-98

2. Morton DL, Wen DR, Wong JH, Economou JS, Cagle LA, Storm FJ, Foshag LJ, Cochran AJ. Technical details of intraoperative lymphatic zapping for early stage melanoma. Arch Surg 1992;127:392-399

3. Nieweg OE, Uren RF, Thompson JF The history of sentinel node biopsy. Cancer J 2015;21:3-6

4. Morton DL, Thompson JF, Cochran AJ, M Mozillo N, Nieweg OE, Roses DF etal. Final trial report of sentinel-node biopsy vs nodal observation in melanoma. New Eng J Med 2014;370:599-609.

5. Balch CM, Thompson JF, Gershenwald JE. Age as a predictor od sentinel node metastasis among patients with localized melanoma: an inverse correlation of melanoma correlation and incidence of sentinel node metastasis among young and old patients. Ann Surg Ocol 2014;21:1075-1081. 